$>$ Le développement de nouveaux modèles animaux et de nouvelles méthodes d'investigation fonctionnelle a radicalement changé notre vision de la physiologie des cellules de la microglie. Ces cellules, qui sont les macrophages résidents $\mathrm{du}$ système nerveux central, apparaissent ainsi comme très dynamiques et capables de réagir rapidement aux modifications de leur environnement. Les recherches actuelles indiquent, en particulier, que ces cellules établissent des contacts physiques avec les synapses neuronales, qu'elles peuvent éliminer ou dont elles peuvent influencer l'activité en libérant des médiateurs spécifiques. Nous présentons ici les arguments expérimentaux qui ont permis de mettre à jour ces interactions, non seulement en conditions pathologiques, mais aussi en conditions physiologiques et, notamment, au cours du développement normal du cerveau. L'existence d'une communication bidirectionnelle entre synapses et microglie éclaire d'un jour nouveau notre compréhension du fonctionnement cérébral et devrait permettre de mieux comprendre les interactions entre système immunitaire et système nerveux. <

Les cellules gliales représentent une population qualitativement hétérogène et quantitativement importante du système nerveux central (SNC). Elles comprennent les astrocytes, les oligodendrocytes, les cellules NG2 (cellules exprimant le protéoglycane NG2) et les cellules microgliales. Ces cellules jouent un rôle important dans I'homéostasie cérébrale en maintenant les gradients ioniques qui permettent la propagation des potentiels d'actions, en pompant les excès de neurotransmetteurs libérés ou encore en assurant une fonction réparatrice lors de lésions ou d'infections [1]. Mais, les 20 dernières années ont vu naître l'idée selon laquelle la glie pouvait prendre une part plus active au traitement de

\section{La microglie \\ Des cellules immunitaires \\ qui sculptent et contrôlent \\ les synapses neuronales}

Étienne Audinat, Isabelle Arnoux

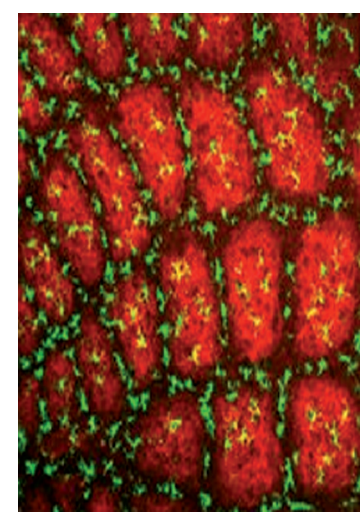

Inserm U1128, Paris, France ; Université Paris Descartes, laboratoire de neurophysiologie et nouvelles microscopies, 45 , rue des Saints-Pères, 75006 Paris, France.

etienne.audinat@parisdescartes.fr

I'information dans le SNC. En effet, les cellules gliales expriment des récepteurs et des transporteurs membranaires leur permettant de suivre l'activité neuronale; elles peuvent réaliser une forme d'intégration de cette activité et, enfin, libérer des médiateurs capables de moduler l'activité neuronale [1]. Dans le cas des astrocytes, le terme de synapse tripartite a été proposé pour rendre compte de cette étroite association fonctionnelle entre les éléments neuronaux pré- et postsynaptiques et les prolongements astrocytaires bordant les synapses [2].

La microglie représente un cas un peu particulier parmi les cellules gliales dans la mesure où elle n'a pas la même origine embryologique [37] $(\rightarrow)$. De fait, ces macrophages résidents du SNC

$\rightarrow$ Voir la synthèse de P. Legendre et $\mathrm{H}$. Le Corronc, page 147 de ce numéro orchestrent les réactions inflammatoires consécutives à des lésions du SNC ou à certaines maladies neurodégénératives, et influencent l'issue de ces pathologies [3, 4]. Mais, des études de plus en plus nombreuses indiquent que la microglie pourrait aussi être impliquée dans des troubles psychiatriques, comme certains comportements compulsifs, syndromes autistiques, démences préséniles, et dans la schizophrénie $[5,6]$. Ces observations suggèrent que ces cellules auraient des fonctions autres que celles attribuées classiquement à des cellules immunitaires, notamment en régulant le développement et l'activité des réseaux neuronaux et synaptiques. Le développement de nouveaux modèles animaux et de nouvelles méthodes d'investigation fonctionnelle indiquent clairement que la microglie est loin d'être inactive dans les conditions physiologiques. De façon analogue aux astrocytes, les cellules microgliales sont sensibles à l'activité neuronale et peuvent influencer le réseau neuronal par différentes formes d'interactions et de voies de signalisation [7]. Nous présentons dans cette revue les données de la littérature récente 


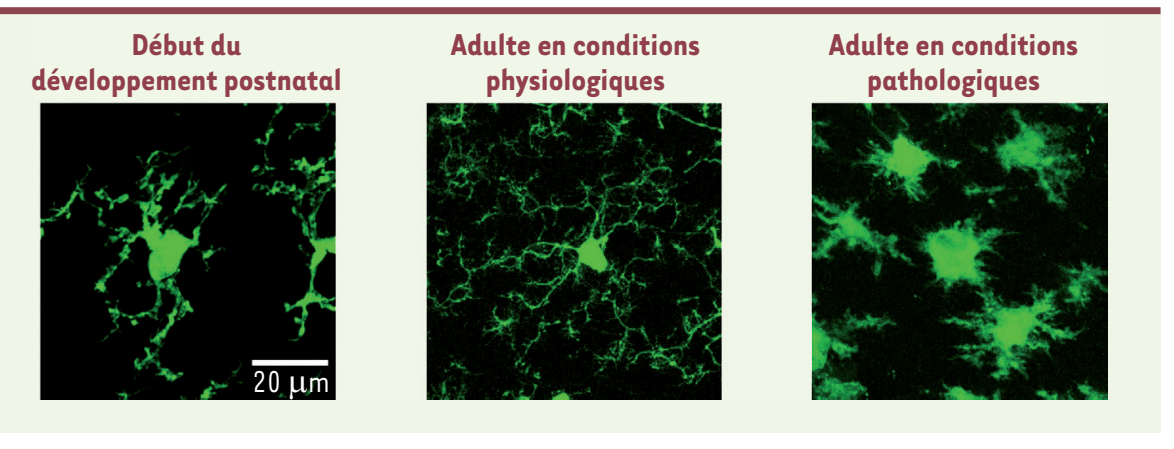

Figure 1. Les différents visages de la microglie. L'utilisation de souris transgéniques dans lesquelles seule la microglie exprime une protéine fluorescente permet de révéler facilement les changements morphologiques de ces cellules au cours du développement normal du cerveau ou dans des conditions pathologiques. Le grossissement est le même pour les trois images.

qui ont amené certains auteurs à proposer la notion de synapse quadripartite [8], dans laquelle la microglie serait le quatrième partenaire.

\section{Activation des cellules microgliales et modifications synaptiques en conditions pathologiques}

En tant que macrophages résidents du SNC, les cellules microgliales ont surtout été étudiées pour leurs rôles en conditions pathologiques. Elles sont très probablement impliquées dans toutes les pathologies cérébrales et sont souvent les premières à être mobilisées, dès l'apparition des premiers signes de la maladie. On sait depuis longtemps que leur morphologie, caractérisée par un petit corps cellulaire et de fins prolongements en conditions physiologiques, évolue vers des formes moins ramifiées avec un corps cellulaire plus grand ou des formes typiquement amiboïdes, lorsqu'elles sont mobilisées dans une pathologie (Figure 1) [37]. Mais ces changements morphologiques ne représentent que la partie visible de l'iceberg. En effet, le recrutement des cellules microgliales en réponse à un message d'alerte s'accompagne d'une modification importante de leur phénotype, appelée activation microgliale, incluant des modifications d'expression de nombreuses protéines, la capacité à libérer de nombreux facteurs pro- et anti-inflammatoires, la capacité à se mouvoir, à phagocyter les débris cellulaires et à proliférer. Cette activation microgliale n'est pas un mécanisme en tout ou rien : elle est progressive et dépend du type de pathologie et du contexte dans lequel elle apparaît. Cette détection fine du changement de l'environnement permet à la microglie d'adopter de nombreux phénotypes dont dépendent les différentes fonctions de ces cellules $[3,4]$.

Parmi les fonctions de la microglie activée, on sait déjà depuis longtemps que ces cellules peuvent prendre la place de synapses sur le corps cellulaire de neurones lésés. Ce processus appelé en anglais synaptic stripping (déshabillage synaptique) a été observé en particulier au niveau des motoneurones du nerf facial après lésion de leurs axones [9]. II ne semble pas se développer dans toutes les pathologies (par exemple, il n'est pas présent dans les maladies à prions) [10], mais a été néanmoins observé dans d'autres régions du SNC que le noyau du nerf facial, telles que le néocortex [11].

La microglie activée peut aussi influencer l'activité synaptique en libérant des médiateurs reconnus par les neurones. L'exemple le mieux caractérisé est celui de la douleur neuropathique et de l'influence de la microglie sur la transmission synaptique inhibitrice dans la moelle épinière. $\varepsilon$ n effet, l'apparition de douleurs consécutives à la lésion de fibres nerveuses périphériques s'accompagne d'une activation microgliale dans la partie de la moelle épinière où sont traitées les informations sensorielles, la corne dorsale (Figure 2A). Cette activation se caractérise en particulier par la mise en jeu d'un récepteur purinergique appelé P2X4 [12, 13, 38] qui est activé par l'adénosine triphosphate (ATP) libérée en excès dans les conditions pathologiques. L'activation de ce récepteur dans la microglie entraîne la production d'un facteur trophique, le BDNF (brain derived neurotrophic factor), qui se lie à son récepteur TrkB (tropomyosin receptor kinase B) localisé à la membrane des neurones de la corne dorsale. La mise en jeu de TrkB va induire un changement dramatique dans le fonctionnement des synapses inhibitrices de la corne dorsale, transformant ces jonctions en synapses excitatrices (Figure 2B). L’hyperexcitabilité du réseau neuronal qui en résulte serait à l'origine des douleurs neuropathiques [14].

Le BDNF n'est qu'un des nombreux médiateurs qui peuvent être libérés par la microglie activée et influencer le devenir des neurones dans les conditions pathologiques (mort, survie), mais aussi de réguler l'activité synaptique via la mise en jeu de voies de signalisation spécifiques, dont certaines font intervenir un troisième partenaire. C'est le cas par exemple du TNF $\alpha$ (tumor necrosis factor $\alpha$ ) qui, dans certaines conditions pathologiques, pourrait exercer son influence sur les neurones de façon indirecte en activant son récepteur TNFRl exprimé par d'autres cellules gliales, les astrocytes [15, 16]. Comme les neurones, les astrocytes ont la capacité de libérer du glutamate qui peut avoir des effets neurotoxiques, mais qui peut aussi moduler l'activité neuronale $[15,17,18]$. Nous verrons plus loin un autre exemple de la mise en jeu de cette triade microglie-astrocyte-neurone. 
A Activation microgliale du côté ipsilatéral à la lésion

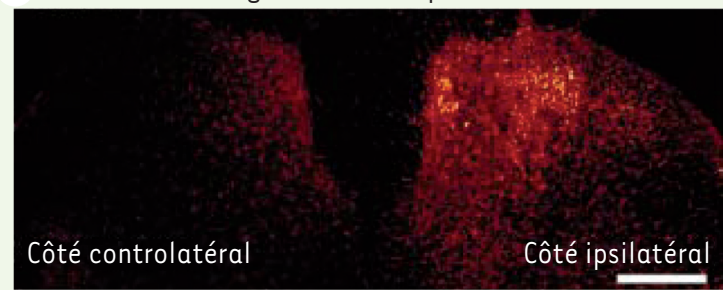

B La microglie activée régule l'inhibition synaptique Microglie Neurone

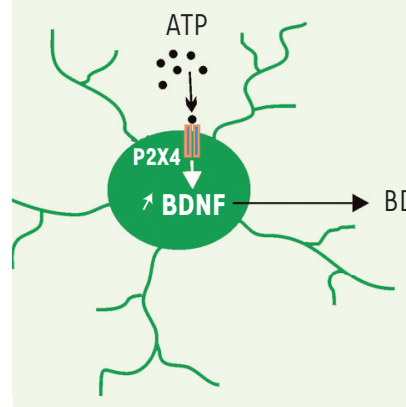
BDNF

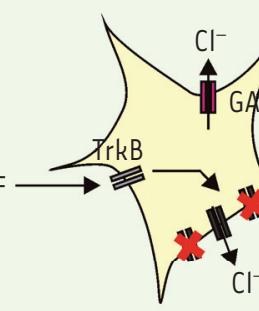

Figure 2. Microglie et douleur neuropathique. A. Illustration de l'activation microgliale dans la corne dorsale du côté ipsilatéral à la lésion périphérique. La microglie est marquée avec un anticorps spécifique (adaptée de [13]). B. Mécanismes liant l'activation microgliale à I'hyperexcitabilité du réseau neuronal de la corne dorsale. La lésion d'un nerf périphérique induit l'activation de la microglie de la corne dorsale. Cette activation se traduit, en particulier, par une expression de novo du récepteur de I'ATP P2X4. La mise en jeu de ce récepteur induit une augmentation de la production de BDNF par la microglie. La liaison du BDNF à son récepteur TrkB exprimé à la membrane des neurones induit une diminution d'expression d'une autre protéine de la membrane des neurones, le transporteur des ions chlore nommé KCC2 (potassium chloride cotransporter 2), qui va entraîner une modification du fonctionnement des synapses inhibitrices. En effet, les récepteurs synaptiques activés par les neurotransmetteurs inhibiteurs, comme le GABA ou la glycine, sont des canaux membranaires perméables au chlore. La baisse d'expression de KCC2 se traduit par une élévation de la concentration de chlore dans le neurone et, donc, une inversion du mouvement des ions chlore lors de la mise en jeu des synapses inhibitrices. Le chlore chargé négativement sort alors de la cellule, au lieu d'y entrer, et ceci tend à dépolariser et donc à exciter les neurones, au lieu de les inhiber. Les synapses inhibitrices sont devenues excitatrices et cette hyperexcitabilité du réseau de la corne dorsale est à l'origine des douleurs neuropathiques.

\section{La microglie en conditions physiologiques : pas si calme que cela !}

Une rupture majeure dans notre compréhension de la physiologie de la microglie a eu lieu il y a un peu moins de 10 ans lorsque deux équipes ont observé le comportement de la microglie directement dans le cerveau de souris saines $[19,20]$. Pour ce faire, les chercheurs ont utilisé des souris transgéniques dans lesquelles seules les cellules microgliales expriment une protéine fluorescente, la GFP (green fluorescent protein). Ils ont placé ces animaux anesthésiés sous un microscope biphotonique permettant l'observation de la fluorescence à l'intérieur du cerveau. Ils ont alors noté que les prolongements fins de la microglie dans le néocortex sont en perpétuel mouvement et explorent un domaine d'environ $100 \mu \mathrm{m}$ de diamètre autour du corps cellulaire immobile de la microglie. Ainsi, contrairement à l'idée qui a prévalu pendant longtemps, la microglie en conditions physiologiques n'est pas quiescente, en attente de l'apparition d'un signal de danger qui l'activerait. Elle est au contraire très dynamique et explore en permanence son environnement en allongeant et en rétractant ses prolongements à une vitesse de l'ordre de quelques micromètres par minute. À quoi peuvent servir ces mouvements? Une hypothèse est qu'ils permettent à la microglie d'aller à la recherche des signaux de danger afin de détecter le plus rapidement possible une rupture de l'homéostasie cérébrale. En effet, ces mouvements, apparemment aléatoires, deviennent tout à fait ordonnés lors de l'apparition d'une lésion: la plupart des prolongements des microglies au voisinage de la lésion convergent alors vers celle-ci et l'entourent rapidement.

Les signaux régulant cette motilité microgliale sont certainement multiples, mais là encore I'ATP joue un rôle important puisqu'il régule la cinétique des mouvements spontanés et orientés vers une lésion. Le récepteur microglial mis en jeu cette fois est le récepteur purinergique P2Y12 [20, 21]. Mais d'autres signaux sont également impliqués, comme l'adénosine qui régule la rétraction des prolongements microgliaux [22] et la dynamique de ces mouvements sensible à l'activité synaptique du réseau neuronal local [23, 24] (même si tous les auteurs ne s'accordent pas sur ce point [25]). Or, dans les conditions physiologiques, la concentration des transmetteurs libérés lors de l'activité synaptique est très contrôlée de façon à éviter que ceux-ci ne s'échappent de la fente synaptique et que leur concentration dans le milieu extracellulaire n'augmente trop de façon prolongée. Si les mouvements de la microglie sont sensibles à l'activité synaptique, il est donc possible que les prolongements microgliaux entrent en contact direct avec les synapses et que la dynamique de ces processus reflète au moins en partie la dynamique des interactions physiques entre microglie et synapses. Cette hypothèse a effectivement pu être confirmée lors d'observations en microscopie biphotonique et électronique d'animaux transgéniques dans lesquels la microglie et les neurones expriment 

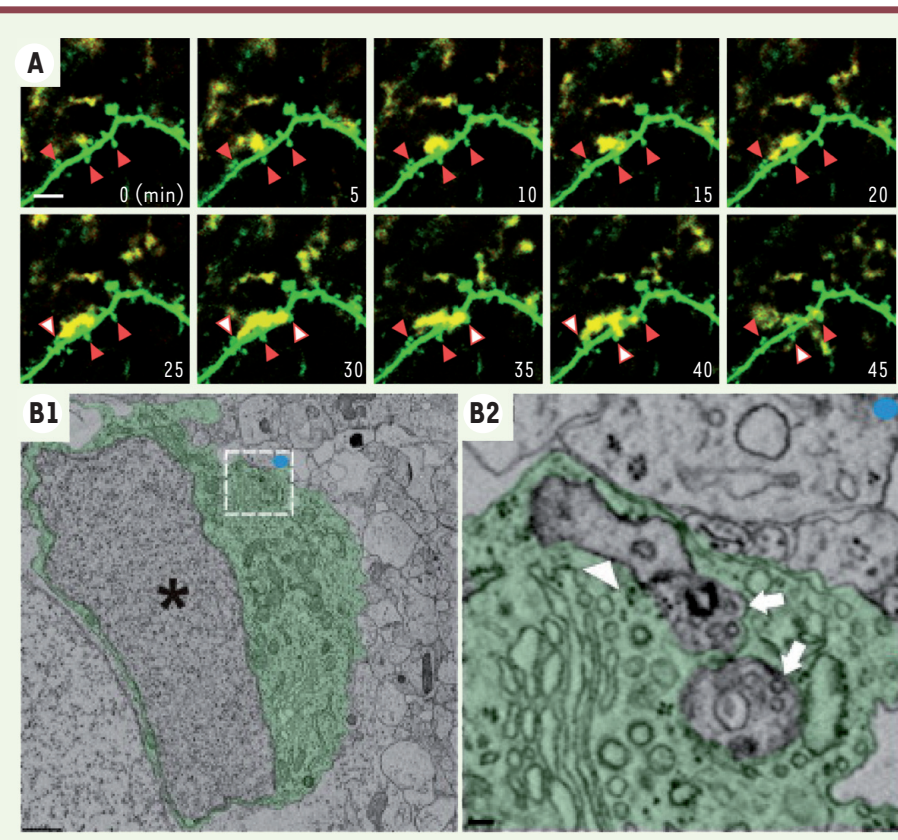

Figure 3. Microglie au contact des synapses. A. Série d'images prises en microscopie biphotonique toutes les 5 minutes dans le cortex d'une souris transgénique adulte exprimant des protéines fluorescentes différentes dans les neurones (vert) et la microglie (jaune). Les flèches indiquent les épines dendritiques postsynaptiques contactées par la microglie. La barre d'échelle représente $5 \mu \mathrm{m}$ (adaptée d'après [26]). B. Clichés de microscopie électronique montrant la présence d'éléments présynaptiques (flèches blanches en B2) et probablement postsynaptiques (tête de flèche blanche en B2) à l'intérieur d'une microglie (verte) dans le thalamus d'une souris âgée de cinq jours. L'astérisque en $B I$ indique le noyau de la cellule microgliale. Le carré blanc en $B I$ indique la zone agrandie présentée en B2. La barre d'échelle en $\mathbf{B 2}$ représente $100 \mathrm{~nm}$ (adaptée d'après [35]).

des protéines fluorescentes distinctes $[23,26]$. Ces expériences ont montré, d'une part, que les prolongements microgliaux en mouvement forment à leur extrémité des contacts directs avec les éléments pré- et postsynaptiques de neurones corticaux (Figure 3A) et, d'autre part, que ces interactions structurelles sont régulées par l'activité neuronale, qui détermine notamment la durée et la fréquence de ces contacts entre microglie et synapses. Les mouvements microgliaux pourraient donc avoir une autre fonction que l'unique recherche de signaux d'alerte et pourraient participer à la régulation de l'activité synaptique. Des résultats en faveur d'une telle hypothèse ont été récemment obtenus dans une étude réalisée sur des larves de poisson zèbre montrant que la microglie est plus fréquemment en contact avec les neurones ayant une forte activité, et que celle-ci diminue à la suite de ce contact [27].

Ainsi, par analogie avec son rôle en conditions pathologiques, la microglie pourrait influencer l'activité synaptique en libérant un certain nombre de médiateurs connus pour moduler l'expression et la fonction de protéines synaptiques. II y a en effet dans la littérature de nombreux exemples de modulation du fonctionnement de synapses dans des conditions non pathologiques par des médiateurs pouvant être libérés par la microglie. Le plus étudié est sans doute encore une fois le TNF $\alpha$ qui, lorsqu'il est appliqué de façon exogène, régule de nombreux aspects de la transmission et de la plasticité synaptiques [28]. Mais, même si la microglie est le pourvoyeur le plus important de TNF $\alpha$ dans le SNC, d'autres sources ne sont pas à exclure et l'implication de la microglie dans ces processus synaptiques n'est donc pas certaine. En revanche, la stimulation spécifique de récepteurs exprimés uniquement par la microglie a permis de confirmer cette hypothèse. Le lipopolysaccharide (LPS) est un composant de la paroi de certaines bactéries qui est classiquement utilisé expérimentalement pour déclencher une activation de la microglie et en étudier les conséquences sur une échelle de temps de plusieurs heures à plusieurs jours. De façon surprenante, I'application de LPS sur des tranches d'hippocampe entraîne une augmentation presque immédiate de la fréquence des courants synaptiques excitateurs spontanés [29]. Les auteurs de cette observation ont disséqué les voies de signalisation impliquées dans cet effet et ont montré que l'activation du récepteur du LPS, TLR4 (toll like receptor 4 ), qui est exprimé uniquement par la microglie en conditions physiologiques, entraîne une libération d'ATP par la microglie, qui active les récepteurs purinergiques P2Yl à la surface des astrocytes. Les astrocytes mobilisés par l'ATP libèrent alors du glutamate, qui se lie à des récepteurs spécifiques, appelés mGluR5, localisés sur les terminaisons présynaptiques des connexions excitatrices et dont il augmente la probabilité de libération de leur neurotransmetteur [29]. Une des surprises de cette étude réside dans la rapidité des effets du LPS qui suggère que, indépendamment de l'activation complète de la microglie induite par ce stimulus, toute voie de signalisation entraînant une libération d'ATP par la microglie a potentiellement la capacité d'augmenter l'excitabilité du réseau neuronal.

D'autres voies de régulation de l'activité synaptique par la microglie en conditions physiologiques ont été identifiées. La fractalkine est une chimiokine exprimée à la membrane des neurones, et son unique récepteur, $C X_{3} C R 1$, n'est exprimé que par la microglie dans le SNC. L'application de fractalkine dans l'hippocampe sain induit une diminution transitoire de l'amplitude des courants synaptiques excitateurs neuronaux. Cet effet passe par une libération microgliale d'adénosine (ou d'ATP hydrolysé en adénosine par des ectonucléotidases extracellulaires) agissant au niveau présynaptique [30]. De façon intéressante, les souris déficientes pour le récepteur $\mathrm{CX}_{3} \mathrm{CRl}$ présentent des déficits dans la transmission et la plasticité synaptique excitatrice glutamatergique ainsi que des déficits cognitifs. Ces anomalies résulteraient d'une production anormalement 
élevée d'interleukine-1 $\beta$, une cytokine pro-inflammatoire qui peut être libérée par la microglie [31]. L'activation constitutive de la voie de la fractalkine empêcherait ainsi une action délétère de la microglie sur le fonctionnement synaptique. II existe au moins deux autres protéines microgliales, CD200R, le récepteur de la glycoprotéine CD200 exprimée par les neurones et d'autres cellules gliales, et DAP12, un adaptateur des récepteurs de type TREM (triggering receptor expressed on myeloid cells), dont la mobilisation ou l'absence affectent la transmission synaptique dans l'hippocampe [32, 33]. Cependant, les mécanismes reliant ces voies microgliales de signalisation à la transmission neuronale restent, à ce jour, inconnus.

Les travaux actuels indiquent donc qu'en conditions physiologiques, la microglie exerce une surveillance permanente des neurones du SNC. Cette surveillance se traduit par la formation de contacts transitoires entre microglie et synapses, et ces interactions physiques semblent influencer le fonctionnement des synapses. Néanmoins, beaucoup de questions restent encore sans réponse. En particulier, comment sont mobilisés les facteurs diffusibles microgliaux influençant l'activité synaptique? Il est en effet plus difficile de mettre en évidence l'intervention de tels médiateurs en conditions physiologiques au cours desquelles leur production est certainement moindre et plus limitée dans le temps que dans des conditions pathologiques. Certains éléments de réponses pourraient émerger de l'étude de la microglie au cours du développement du SNC pendant lequel des changements synaptiques structuraux et fonctionnels massifs dépendant de l'activité semblent nécessiter une intervention de la microglie.

\section{La microglie au cours du développement postnatal du SNC : un sculpteur des synapses en formation}

Au cours du développement normal du SNC, un nombre important de neurones sont éliminés par apoptose et la microglie régule ce processus $\rightarrow$ Voir la synthèse de P. Legendre et H. Le Corronc, page 147 de ce numéro $[37](\rightarrow)$.

Mais, il y a aussi une production de synapses en excès qui sont éliminées par des mécanismes dépendant en partie de l'activité neuronale lors de la maturation des réseaux synaptiques. Des observations récentes indiquent que, durant le développement postnatal de l'hippocampe et du thalamus de la souris, des éléments pré- et postsynaptiques ont été observés à l'intérieur de cellules microgliales (Figure 3B). Cela suggère que celles-ci sont impliquées dans la phagocytose de synapses surnuméraires devant être éliminées [34, 35]. Dans le thalamus en développement, la phagocytose des terminaisons des fibres rétiniennes est dépendante de l'activité neuronale et met en jeu des éléments du système du complément, mieux connu pour son rôle dans le système immunitaire. II semble que les synapses « faibles » devant être éliminées sont étiquetées par l'expression de la composante C3 du complément, qui agit comme un signal «mange moi » (eat me) sur la microglie, qui est la seule composante du SNC à exprimer le récepteur de C3. Chez les animaux invalidés pour C3 ou son récepteur microglial C3R, la phagocytose des terminaisons rétiniennes par la microglie est diminuée et la connectivité synaptique de cette voie rétino-thalamique ne subit pas

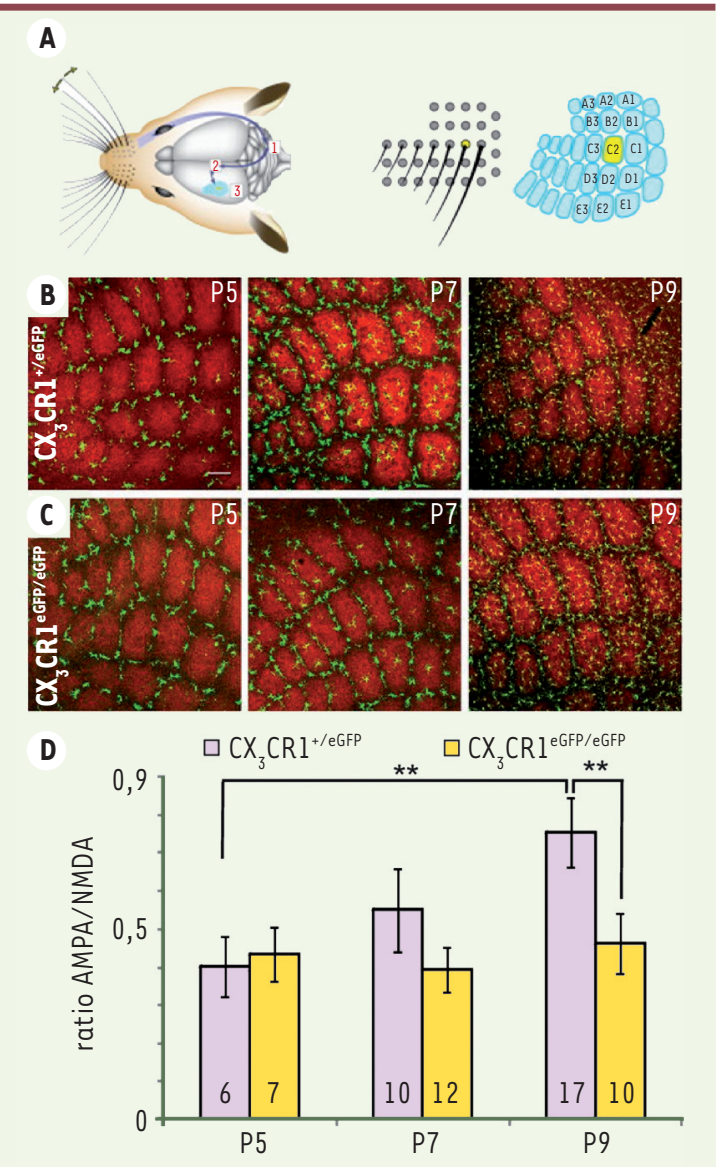

Figure 4. Microglie et maturation fonctionnelle des synapses. A. Schéma du système sensoriel des vibrisses chez les rongeurs et correspondance entre la disposition des vibrisses du museau et les tonneaux dans la couche 4 du cortex somato-sensoriel (adaptée d'après [40]). B. Évolution de la distribution de la microglie (vert) dans le champ des tonneaux de la couche 4 $\mathrm{du}$ cortex entre le cinquième et le neuvième jour postnatal chez des souris hétérozygotes pour le récepteur microglial de la fractalkine $\left(\mathrm{CX}_{3} \mathrm{CRl^{+/eGFP }}\right)$. Notez qu'au cinquième jour, la microglie est exclue des zones synaptiques au centre des tonneaux (rouge, marquage des axones thalamiques) et qu'au septième jour postnatal, elle a commencé à envahir ces zones. La barre d'échelle représente $100 \mu \mathrm{m}$. C. Même expérience réalisée avec des souris invalidées pour $\mathrm{CX}_{3} C R 1^{\text {eGFP/eGFP. Notez }}$ qu'au septième jour postnatal, la microglie n'a pratiquement pas commencé à envahir les tonneaux. D. Évolution relative des courants synaptiques portés par les deux types de récepteurs du glutamate, les récepteurs AMPA ( $\alpha$-amino-3-hydroxy-5methyl-4-isoxazolepropionic acid) et NMDA ( $N$-methyl-Daspartate receptor), exprimés aux synapses thalamo-corticales entre le cinquième et le neuvième jour postnatal chez les souris hétérozygotes ou invalidées pour CX3CR1. Notez le déficit d'évolution chez les souris invalidées pour CX3CRl, qui constitue un défaut de maturation fonctionnelle de ces synapses (adaptée d'après [36]). 
une maturation normale [35]. La disponibilité de la microglie aux sites d'élimination synaptique est bien entendu un facteur limitant pour que cette phagocytose ait lieu. Dans l'hippocampe d'animaux invalidés pour le récepteur $\mathrm{CX}_{3} \mathrm{CRl}$ de la fractalkine, l'augmentation de la densité microgliale est retardée pendant les premières semaines de vie. Ce déficit transitoire du nombre de microglies s'accompagne d'un plus grand nombre d'épines dendritiques sur les neurones, suggérant un déficit d'élimination des synapses surnuméraires. De façon intéressante, ces anomalies structurelles s'accompagnent d'un retard de maturation des propriétés fonctionnelles du réseau neuronal [34]. Cependant, le lien entre phagocytose microgliale et maturation fonctionnelle des synapses n'est pas clairement établi, et d'autres fonctions microgliales pourraient expliquer ces observations.

Le rôle de la microglie sur le développement des circuits du cortex somato-sensoriel des rongeurs a été étudié au niveau de structures anatomiques très particulières appelées tonneaux. Le centre de ces tonneaux est constitué par les fibres issues du thalamus, une structure sous-corticale relayant les informations sensorielles issues des vibrisses du museau, véritables organes sensoriels des rongeurs [39]. Au centre des tonneaux, les fibres thalamiques font des synapses sur les dendrites des neurones du cortex dont les corps cellulaires sont localisés à la périphérie des tonneaux. À chaque vibrisse correspond un seul tonneau dans le cortex (Figure $3 A$ ). Cette représentation corticale des vibrisses se met en place à partir du troisième jour postnatal, et les propriétés fonctionnelles des synapses thalamo-corticales évoluent de façon importante entre la première et la seconde semaine postnatale. La distribution de la microglie pendant ces étapes de formation des tonneaux et de maturation synaptique suit un patron très stéréotypé. Jusqu'au cinquième jour postnatal, la microglie reste à la périphérie des tonneaux et est exclue des sites synaptiques qu'elle commence à envahir uniquement à partir des sixième et septième jours (Figure 4B). Ce recrutement microglial au niveau des synapses dépend de la fractalkine qui est exprimée de façon transitoire au centre des tonneaux. Chez les animaux invalidés pour le récepteur $\mathrm{CX}_{3} \mathrm{CRl}$, le recrutement microglial est retardé d'au moins deux jours (Figure 4C). Ce retard de recrutement de la microglie aux sites des synapses thalamo-corticales s'accompagne d'un retard de maturation des propriétés de ces synapses qui se caractérise en particulier par un défaut dans l'expression fonctionnelle des récepteurs postsynaptiques du glutamate [36]. Ces observations suggèrent donc que la microglie, en colonisant le centre des tonneaux, émet un signal influençant l'expression ou la disponibilité des récepteurs du glutamate aux synapses. L'identité de ce signal doit encore être déterminée, mais ces observations soulignent que la microglie influence la maturation fonctionnelle des circuits synaptiques durant le développement normal du SNC.

\section{Conclusion}

L'ensemble de ces travaux souligne l'extraordinaire plasticité des cellules microgliales, dont le phénotype et les fonctions s'adaptent rapidement à l'environnement. L'existence d'une motilité permanente de la microglie en direction des synapses dans les conditions physiologiques est une découverte fascinante. Son étude pourrait avoir d'importantes conséquences sur notre conception du contrôle de l'activité synaptique, notamment lors des changements d'état du système (veille-sommeil, stress, etc.). Ainsi, si la synapse quadripartite est une réalité morphologique, nous sommes encore loin d'en comprendre le fonctionnement, et les spécificités passionnantes de la microglie devraient nous occuper encore un certain temps. $\diamond$

\section{SUMMARY}

Microglia: immune cells sculpting and controlling neuronal synapses

The development of new animal models and functional analysis methods has dramatically changed our understanding of the physiology of microglial cells. These cells which are the resident macrophages of central nervous system have the ability to adapt rapidly to subtle changes of their environment. Recent findings indicate in particular that they can establish contacts with neuronal synapses that they can eliminate and modulate by releasing specific mediators. Here we review the experimental observations that have revealed the occurrence of these interactions not only in pathological conditions but also in the healthy brain and in particular during normal brain development. The discovery of bi-directional communications between synapses and microglia sheds a new light on our understanding of brain functioning and should allow a better understanding of brain functioning and of interactions between immune and nervous systems. $\diamond$

\section{LIENS D'INTÉRÊT}

Les auteurs déclarent n'avoir aucun lien d'intérêt concernant les données publiées dans cet article.

\section{REMERCIEMENTS}

Nous remercions Céline Bidoret pour son aide sur l'étude morphologique et Maki Hoshiko pour sa contribution à l'ensemble du projet microglie et développement. Isabelle Arnoux a bénéficié d'allocations du ministère de l'Enseignement supérieur et de la Recherche et de la Fondation pour la recherche médicale (FDT20130928365). Les images confocales des Figures 1-4 ont été acquises sur la plateforme du centre universitaire des Saints-Pères. L'équipe d'Étienne Audinat est membre de l'École des neurosciences de Paris (ENP) et est soutenue par l'Inserm, le CNRS et l'Agence nationale de la recherche (ANR 2010 BLAN 141901 ).

\section{RÉFÉRENCES}

1. Verkhratsky A, Butt A. Glial neurobiology: a text book. New York : John Wiley and Sons Ltd, 2007.

2. Perea G, Navarrete M, Araque A. Tripartite synapses: astrocytes process and control synaptic information. Trends Neurosci 2009 ; 32 : 421-31. 


\section{RéFÉRENCES}

3. Hanisch UK, Kettenmann H. Microglia: active sensor and versatile effector cells in the normal and pathologic brain. Nat Neurosci $2007 ; 10: 1387-94$.

4. Ransohoff RM, Perry VH. Microglial physiology: unique stimuli, specialized responses. Annu Rev Immunol $2009 ; 27: 119-45$.

5. Bechade C, Cantaut-Belarif $y$, Bessis A. Microglial control of neuronal activity. Front Cell Neurosci $2013 ; 7: 32$

6. Blank T, Prinz M. Microglia as modulators of cognition and neuropsychiatric disorders. Glia 2013 ; $61: 62-70$.

7. Kettenmann H, Hanisch UK, Noda M, Verkhratsky A. Physiology of microglia. Physiol Rev $2011 ; 91$ : 461-553.

8. Schafer DP, Lehrman EK, Stevens B. The quad-partite synapse: microglia-synapse interactions in the developing and mature CNS. Glia $2013 ; 61: 24-36$.

9. Blinzinger K, Kreutzberg $G$. Displacement of synaptic terminals from regenerating motoneurons by microglial cells. Z Zellforsch Mikrosk Anat 1968; 85 : 145-57.

10. Siskova Z, Page A, O'Connor V, Perry VH. Degenerating synaptic boutons in prion disease: microglia activation without synaptic stripping. Am J Pathol $2009 ; 175: 1610-21$.

11. Trapp BD, Wujek JR, Criste GA, et al. Evidence for synaptic stripping by cortical microglia. Glia $2007 ; 55: 360-8$

12. Ulmann L, Hatcher JP, Hughes JP, et al. Up-regulation of $P 2 X 4$ receptors in spinal microglia after peripheral nerve injury mediates BDNF release and neuropathic pain. J Neurosci $2008 ; 28$ : 11263-8

13. Tsuda M, Shigemoto-Mogami Y, Koizumi S, et al. P2X4 receptors induced in spinal microglia gate tactile allodynia after nerve injury. Nature $2003 ; 424: 778-83$

14. Coull JA, Beggs $S$, Boudreau D, et al. BDNF from microglia causes the shift in neuronal anion gradient underlying neuropathic pain. Nature $2005 ; 438$ : 1017-21.

15. Bezzi P, Domercq M, Brambilla L, et al. CXCR4-activated astrocyte glutamate release via TNFalpha: amplification by microglia triggers neurotoxicity. Nat Neurosci $2001 ; 4: 702-10$.

16. Domercq M, Brambilla L, Pilati $\varepsilon$, et al. P2Yl receptor-evoked glutamate exocytosis from astrocytes: control by tumor necrosis factor-alpha and prostaglandins. J Biol Chem 2006; 281 : 30684-96.

17. Perea G, Araque A. GLIA modulates synaptic transmission. Brain Res Rev 2010 ; 63 : 93-102.

18. Angulo MC, Le MK, Kozlov AS, et al. GABA, a forgotten gliotransmitter. Prog Neurobiol 2008 ; 86 : 297-303.

19. Nimmerjahn A, Kirchhoff $F$, Helmchen F. Resting microglial cells are highly dynamic surveillants of brain parenchyma in vivo. Science $2005 ; 308: 1314-8$.

20. Davalos D, Grutzendler J, Yang $G$, et al. ATP mediates rapid microglial response to local brain injury in vivo. Nat Neurosci $2005 ; 8: 752-8$.

21. Haynes SE, Hollopeter G, Yang G, et al. The P2Y12 receptor regulates microglial activation by extracellular nucleotides. Nat Neurosci $2006 ; 9$ : 1512-9.

22. Orr AG, Orr AL, Li XJ, et al. Adenosine $A(2 A)$ receptor mediates microglial process retraction. Nat Neurosci $2009 ; 12: 872-8$.

23. Wake $\mathrm{H}$, Moorhouse AJ, Jinno S, et al. Resting microglia directly monitor the functional state of synapses in vivo and determine the fate of ischemic terminals. J Neurosci 2009 ; 29 : 3974-80.
24. Fontainhas AM, Wang M, Liang KJ, et al. Microglial morphology and dynamic behavior is regulated by ionotropic glutamatergic and GABAergic neurotransmission. PLoS One $2011 ; 6$ : el5973.

25. Wu LJ, Zhuo M. Resting microglial motility is independent of synaptic plasticity in mammalian brain. J Neurophysiol 2008 ; 99 : 2026-32.

26. Tremblay ME, Lowery RL, Majewska AK. Microglial interactions with synapses are modulated by visual experience. PLoS Biol $2010 ; 8$ : el000527.

27. Li Y, Du XF, Liu CS, et al. Reciprocal regulation between resting microglial dynamics and neuronal activity in vivo. Dev Cell 2012;23:1189-202.

28. Santello M, Volterra A. TNFalpha in synaptic function: switching gears. Trends Neurosci $2012 ; 35: 638-47$

29. Pascual 0 , Ben AS, Rostaing P, et al. Microglia activation triggers astrocytemediated modulation of excitatory neurotransmission. Proc Natl Acad Sci USA 2012 ; 109 : ع197-205.

30. Piccinin S, Di AS, Piccioni A, et al. CX3CLl-induced modulation at CAl synapses reveals multiple mechanisms of EPSC modulation involving adenosine receptor subtypes. J Neuroimmunol $2010 ; 224: 85-92$.

31. Rogers JT, Morganti JM, Bachstetter AD, et al. CX3CRI deficiency leads to impairment of hippocampal cognitive function and synaptic plasticity. J Neurosci $2011 ; 31: 16241-50$.

32. Costello DA, Lyons $A$, Denieffe $S$, et al. Long term potentiation is impaired in membrane glycoprotein CD200-deficient mice: a role for Toll-like receptor activation. J Biol Chem 2011; 286 : 34722-32.

33. Roumier A, Bechade C, Poncer JC, et al. Impaired synaptic function in the microglial KARAP/DAP12-deficient mouse. J Neurosci 2004 ; 24 : 11421-8.

34. Paolicelli RC, Bolasco G, Pagani F, et al. Synaptic pruning by microglia is necessary for normal brain development. Science $2011 ; 333: 1456-8$.

35. Schafer DP, Lehrman EK, Kautzman AG, et al. Microglia sculpt postnatal neural circuits in an activity and complement-dependent manner. Neuron $2012 ; 74: 691-705$

36. Hoshiko M, Arnoux I, Avignone $\varepsilon$, et al. Deficiency of the microglial receptor CX3CR1 impairs postnatal functional development of thalamocortical synapses in the barrel cortex. J Neurosci 2012 ; 32 : 15106-11.

37. Legendre $P$, Le Corronc $H$. Cellules microgliales et développement du système nerveux central chez l'embryon. Med Sci (Paris) 2014 ; 30 : 147-52.

38. Pochet S, Seil M, El Ouaaliti M, Dehaye JP. P2X4 ou P2X7. Lequel de ces deuX récepteurs nous fera saliver? Med Sci (Paris) 2013 ; 29 : 509-14.

39. Estebanez L, Destexhe A, El-Boustani S, Shulz DE. Ce que les vibrissent disent au cerveau tactile. Med Sci (Paris) 2014 ; $30: 93-8$.

40. Petersen CC. The functional organization of the barrel cortex. Neuron 2007 $56: 339-55$.

\section{TIRÉS À PART}

\section{ع. Audinat}

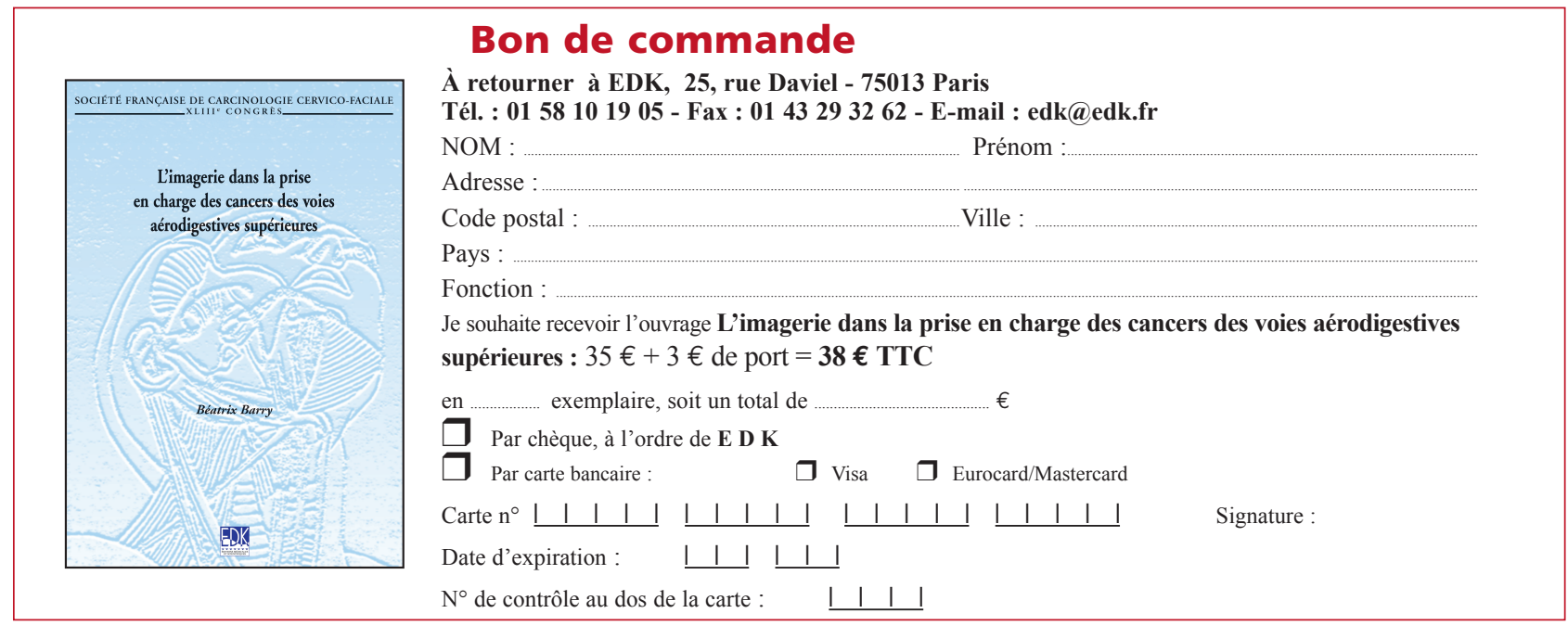

